\title{
Activity of Human Dihydrolipoamide Dehydrogenase Is Reduced by Mutation at Threonine-44 of FAD-binding Region to Valine
}

\author{
Hakjung Kim* \\ Department of Chemistry, College of Natural Science, Daegu University, Kyoungsan 712-714, Korea
}

Received 3 May 2002, Accepted 18 June 2002

\begin{abstract}
Dihydrolipoamide dehydrogenase (E3) is a member of the pyridine nucleotide-disulfide oxidoreductase family. Thr residues are highly conserved. They are at the active site disulfide-bond regions of most E3s and other oxidoreductases,. The crystal structure of Azotobacter vinelandii $\mathbf{E 3}$ suggests that the hydroxyl group of Thr that are involved in the FAD binding interact with the adenosine phosphate of FAD. However, several prokaryotic E3s have Val instead of Thr. To investigate the meaning and importance of the Thr conservation in many E3s, the corresponding residue, Thr-44, in human E3 was substituted to Val by site-directed mutagenesis. The mutant's E3 activity showed about a 2.2-fold decrease. Its UV-visible and fluorescence spectra indicated that the mutant might have a slightly different microenvironment at the FAD-binding region.
\end{abstract}

Keywords: Dihydrolipoamide dehydrogenase, Active site disulfide bond, Pyridine nucleotide-disulfide oxidoreductases, Site-directed mutagenesis, Structural homology

\section{Introduction}

Dihydrolipoamide dehydrogenase (E3) (dihydrolipoamide: $\mathrm{NAD}^{+}$oxidoreductase; EC 1.8.1.4) is a common component in three $\alpha$-keto acid dehydrogenase complexes (pyruvate, $\alpha$ ketoglutarate, and branched-chain $\alpha$-keto acid dehydrogenase complexes) (Reed, 1974) and the glycine cleavage system (Walker and Oliver, 1986). It catalyzes the reoxidation of the dihydrolipoyl prosthetic group that is attached to the lysyl residue(s) of the acyltransferase components of the three $\alpha$ keto acid dehydrogenase complexes, as well as to the hydrogen-carrier protein of the glycine cleavage system. It is a homodimeric flavoprotein that contains one FAD as a prosthetic group at each subunit. Each human E3 subunit

*To whom correspondence should be addressed.

Tel: 82-53-850-6447; Fax: 82-53-850-6449

E-mail: hjkim@taegu.ac.kr. consists of 474 amino acids with a molecular mass of 50,216 daltons, calculated from the primary amino acid sequence (Pons et al., 1988).

E3 belongs to the pyridine nucleotide-disulfide oxidoreductase family, which also includes glutathione reductase, mercuric reductase, thioredoxin reductase, and trypanothione reductase (William, 1976). From comparison studies of the E3s structures and glutathione reductases from several sources, it becomes possible to predict the amino acid residues that are important in the human E3 function (Carothers et al., 1989; Thekkumkara et al., 1989). The structure of the human E3 active site has been proposed (Jentoft et al., 1992) on the basis of the three-dimensional structures of the human glutathione reductase (Thieme et al., 1981) and Azotobacter vinelandii E3 (Schierbeek et al., 1989). Several site-specifically modified human E3s were made and characterized, based on these studies (Kim and Patel, 1992; Kim, 1999a; Kim, 1999b).

The catalytic mechanisms of pyridine nucleotide-disulfide oxidoreductases are similar and the disulfide bond at the active site plays a critical role in the catalysis. Cys- 45 and Cys-50 have been identified as the cysteines that form a disulfide bond at the active site of human E3 (Jentoft et al., 1992). The bond should be broken during the catalysis to form an intermediate with the substrate. The disulfide bond of Azotobacter vinelandii $\mathrm{E} 3$ shows an unusual structure where the distance between the $\mathrm{C}^{\alpha}-\mathrm{C}^{\alpha}$ distance is relatively shorter than the normal value (Mattevi et al., 1991). The geometric strain may be necessary for the breakage of the disulfide bond during catalysis. The residues around the active site disulfide bonds of most pyridine nucleotide-disulfide oxidoreductases are highly conserved. It may be conceivable that the residues around the disulfide bond are highly conserved in order to form and keep the strain. The consensus sequence for the active site disulfide bond region of $34 \mathrm{E} 3 \mathrm{~s}$, which have known primary structures from various sources, was searched. The consensus sequence of GG(TV)CLN(VX)GCIP was suggested (Kim, 2001).

The crystal structure of Azotobacter vinelandii E3 revealed 
that the Thr-47 hydroxyl group interacts with the adenosine phosphate of FAD (Mattevi et al., 1991). The Thr residue is conserved in most E3s. It is also highly conserved in other homologous enzymes of the pyridine nucleotide-disulfide oxidoreductase family. However, several prokaryotic E3s have Val or Ile at the site instead of Thr. The corresponding residue, Thr-44, in human E3 was substituted to Val by site-directed mutagenesis. The E3 mutant was expressed in Escherichia coli and highly purified using affinity chromatography. The mutant showed approximately 45\% E3 activity and slightly different UV-visible and fluorescence spectra, which indicates small changes in the FAD environment.

\section{Materials and Methods}

Materials. The electrophoresis reagents, imidazole, iminodiacetic acid sepharose $6 \mathrm{~B}$, lipoamide and $\mathrm{NAD}^{+}$, were from the Sigma Chemical Co. (St. Louis, USA). Dihydrolipoamide was synthesized by the reduction of lipoamide using sodium borohydride. Isopropyl$\beta$-D-thiogalactopyranoside (IPTG) was from POSCOCHEM R \& D Center (Pohang, Korea). The E. coli XL1-Blue that contained the human E3 expression vector pPROEX-1 : E3 was a generous gift from Dr. Mulchand S. Patel of the State University of New York at Buffalo. The PCR premix, primers, and dNTP were from Bioneer (Cheongwon, Korea).

Site-directed mutagenesis and construction of the human E3 mutant expression vector pPROEX-1:E3 (T-44->V). A polymerase chain reaction (PCR) was performed with a 5' primer (5'-TTACGATATCCCAACGACCG-3'), 3' primer (5'-GCAATTC CACCTGTTAAAGC-3'), 5' mutagenic primer (5'-AATGAAACA CTTGGTGGAGTATGCTTGAATGTTGGTTGTA-3': mismatched bases are underlined), and 3' mutagenic primer (5'-TACAACCAA CATTCAAGCATACTCCACCAAGTGTTTCATT-3': mismatched bases are underlined). The reaction was carried out with a Bioneer PCR premix in a programmable PCR machine using the human E3 expression vector pPROEX-1: E3 as a template (Cho, 2000; Kim et al., 2001; Lee and Cho, 2001). After denaturation of the template DNA at $94^{\circ} \mathrm{C}$ for $5 \mathrm{~min}, 35$ rounds of temperature cycling were performed at $94^{\circ} \mathrm{C}$ for $30 \mathrm{sec}, 45^{\circ} \mathrm{C}$ for $30 \mathrm{sec}, 72^{\circ} \mathrm{C}$ for $30 \mathrm{sec}$, followed by a final 5 -min incubation at $72^{\circ} \mathrm{C}$. PCR generated a 350 bp DNA fragment that contained a human E3 sequence of which a codon (ACA) for Thr-44 was substituted to a codon (GTA) for Val. The fragment was digested with NarI and $\mathrm{XbaI}$ to generate a 219 bp NarI/XbaI fragment. The NarI/XbaI fragment was ligated with pPROEX-1 : E3 of which NarI and XbaI digestions removed the corresponding normal $N a r \mathrm{I} / \mathrm{X} b a \mathrm{I}$ fragment. The ligation resulted in the construction of the human E3 mutant expression vector pPROEX-1 : E3(T-44->V). The mutation was confirmed by DNA sequencing.

Expression and purification of the human E3 mutant. One ml of an overnight culture of $E$. coli XL1-Blue that contained the mutant expression vector was used to inoculate $200 \mathrm{ml}$ of an LB medium that contained ampicillin $(100 \mathrm{~g} / \mathrm{ml})$. The cells were grown at $37^{\circ} \mathrm{C}$ to an absorbance of 0.7 at $595 \mathrm{~nm}$. IPTG was added to the final concentration of $1 \mathrm{mM}$. The growing temperature was shifted to $30^{\circ} \mathrm{C}$, and the cells were allowed to grow overnight. The overnight culture was harvested by centrifugation at $4000 \mathrm{~g}$ for $5 \mathrm{~min}$. The cell pellets were washed with a $50 \mathrm{mM}$ potassium phosphate buffer ( $\mathrm{pH}$ 8.0) that contained $100 \mathrm{mM} \mathrm{NaCl}$ (Binding buffer), then recollected by centrifugation at $4000 \mathrm{~g}$ for $5 \mathrm{~min}$. They were resuspended in $10 \mathrm{ml}$ of a binding buffer. They were lysed by a sonication treatment and centrifuged at $10,000 \mathrm{~g}$ for $20 \mathrm{~min}$. The supernatant was loaded on a nickel iminodiacetic acid sepharose $6 \mathrm{~B}$ column. The column was washed with 10 column volumes of a binding buffer, then with the same volume of a binding buffer that contained $150 \mathrm{mM}$ imidazole. The E3 mutant was eluted with a binding buffer that contained $500 \mathrm{mM}$ imidazole. The E3 mutant was dialyzed twice against a $10 \mathrm{mM}$ potassium phosphate buffer (pH 7.5) to remove imidazole.

E3 assay and spectroscopic study. E3 activity was assayed at $37^{\circ} \mathrm{C}$ in a $50 \mathrm{mM}$ potassium phosphate buffer ( $\mathrm{pH}$ 8.0) that contained $1.5 \mathrm{mM}$ EDTA (Kim and Patel, 1992). The activity was recorded spectrophotometrically by observing the reduction of $\mathrm{NAD}^{+}$at $340 \mathrm{~nm}$ with a Shimazu UV $160 \mathrm{~A}$ recording spectrophotometer. One unit of activity is defined as $1 \mathrm{~mol}$ of $\mathrm{NAD}^{+}$ reduced per $\mathrm{min} / \mathrm{mg}$ of protein at $37^{\circ} \mathrm{C}$.

The UV-visible absorption spectra were recorded from $250 \mathrm{~nm}$ to $550 \mathrm{~nm}$ using the same spectrophotometer. The fluorescence spectra were recorded using a Fluoromax spectrofluorometer (Industries, Inc., Edison, USA). The samples were excited at $296 \mathrm{~nm}$ and the emissions were recorded from $310 \mathrm{~nm}$ to $580 \mathrm{~nm}$. The data were transferred to an ASCII file, and the spectra were drawn using the MicroCal Origin Program (Photon Technology International, South Brunswick, USA).

\section{Results and Discussion}

Table 1 shows a comparison of the amino acid sequences around the active site disulfide bond regions of human E3 and the corresponding residues of other E3s from several sources. The 11 amino acids of the active site disulfide bond region of E3 are highly conserved. Among 11 amino acid residues, 6 residues are absolutely conserved. They include the 1 st and 2nd Gly residues, the 4th Cys residue, the 8th Gly residue, the 9th Cys residue, and the 11th Pro residue. There are three highly-conserved residues, which are the 5th Leu, the 6th Asn, and the 10th Ile residues. There are two relatively lessconserved residues, the $3 \mathrm{rd}$ and 7 th residues. The main amino acid for the 3 rd site is Thr (68\% conservation) (Table 1). The minor amino acids are Val (26\%) and Ile (6\%). It is interesting that the Thr residue is conserved in most eukaryotic E3s and many prokaryotic E3s, while several prokaryotic E3s (including E. coli E3) have Val or Ile instead of Thr. The Thr residue is also highly-conserved in the active site disulfide bond regions of the other homologous enzymes of the pyridine nucleotide-disulfide oxidoreductase family, such as glutathione reductases, mercuric reductases, and trypanothione reductases. It would be interesting to know the structural and functional effects of Thr to Val substitutions in 
Table 1. Comparison of amino acid sequences around the disulfide bond at the active site of human E3 and the corresponding residues of other E3s from several sources. Bold letters highlight the Thr residue in human E3 as well as corresponding residues in other E3s.

\begin{tabular}{|c|c|}
\hline Source & Amino acid sequence \\
\hline Homo sapiens (Human) & GGTCLNVGCIP \\
\hline Sus scrofa (Pig) & GGTCLNVGCIP \\
\hline Canis familiaris (Dog) & GGTCLNVGCIP \\
\hline Mus musculus (Mouse) & GGTCLNVGCIP \\
\hline Trypanosoma brucei & GGTCLNVGCIP \\
\hline Trypanosoma cruzi & GGTCLNVGCIP \\
\hline Pisum sativum (Garden pea) & GGTCLNVGCIP \\
\hline Manduca sexta (Tobacco hawk moth) & GGTCLNVGCIP \\
\hline Saccharomyces cerevisiae (Yeast) & GGTCLNVGCIP \\
\hline Schizosaccharomyces pombe (Fission Yeast) & GGTCLNVGCIP \\
\hline Pseudomonas putida (lpdg) & GGTCLNVGCIP \\
\hline Pseudomonas putida (lpd3) & GGTCLNVGCMP \\
\hline Pseudomonas fluorescens & GGTCLNVGCIP \\
\hline Azotobacter vinelandii & GGTCLNVGCIP \\
\hline Rhodobacter capsulatus & GGTCLNVGCIP \\
\hline Alcaligenes eutrophus & GGTCLNVGCIP \\
\hline Pseudomonas putida (lpdv) & GGTCLNIGCIP \\
\hline Chlamydia trachomatis & GGTCLNRGCIP \\
\hline Chlamydia pneumoniae & GGTCLNRGCIP \\
\hline Halobacterium volcanii & GGTCLNYGCIP \\
\hline Bacillus subtilis (acol) & GGTCLNEGCIP \\
\hline Bacillus subtilis (bfmbc) & GGTCLHKGCIP \\
\hline Synechocytis sp. & GGTCVNRGCIP \\
\hline Escherichia coli & GGVCLNVGCIP \\
\hline Bacillus stearothermophilus & GGVCLNVGCIP \\
\hline Bacillus subtilis & GGVCLNVGCIP \\
\hline Staphylococcus aureus & GGVCLNVGCIP \\
\hline Vibrio parahaemolyticus & GGVCLNVGCIP \\
\hline Mycoplasma pneumoniae & GGVCLNVGCIP \\
\hline Mycoplasma genitalium & GGVCLNVGCIP \\
\hline Haemophilus influenzae & GGVCLNVGCIP \\
\hline Chlorobium vibrioforme & GGVCVNWGCIP \\
\hline Acholeplasma laidlawii & GGICLNHGCIP \\
\hline Zymomonas mobilis & GGICLNWGCIP \\
\hline
\end{tabular}

the human E3 in order to discover the reason for the Thr conservation in human E3, as well as many other E3s.

To investigate the meaning and importance of the Thr conservation in human E3, the corresponding residue, Thr-44, in human E3 was substituted for Val by site-directed mutagenesis. The site-directed mutagenesis and construction of the human E3 mutant expression vector pPROEX-1:E3 (T44->V) were performed, as described in Materials and Methods. The mutation was confirmed by DNA sequencing. The expression and purification of the mutant were performed, as described in Materials and Methods.

An E3 assay was performed at $37^{\circ} \mathrm{C}$. The mutant E3

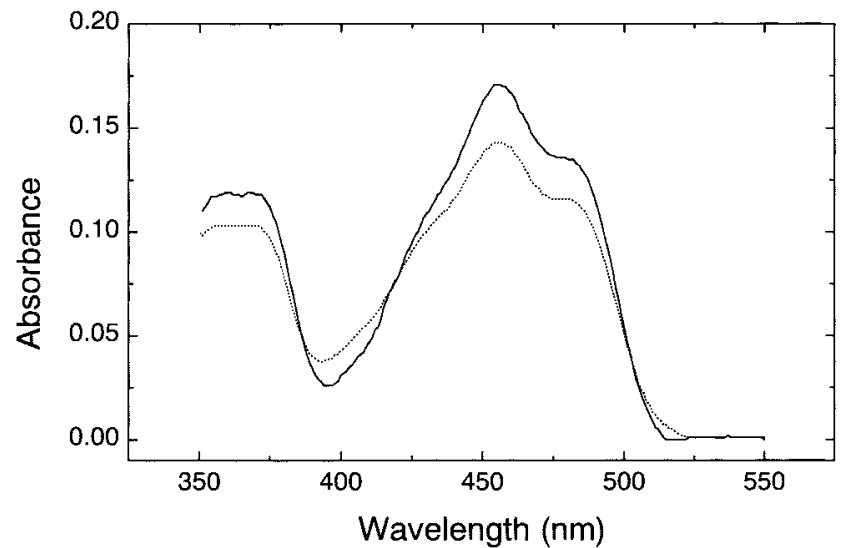

Fig. 1. UV-visible spectra of the mutant $(15.1 \mu \mathrm{M}$, solid line) and wild-type $(12.5 \mu \mathrm{M}$, dot line) recombinant human E3s. The spectra were recorded using a Shimazu UV 160A spectrophotometer. The data from $350 \mathrm{~nm}$ to $550 \mathrm{~nm}$ were transferred to an ASCII file. The spectra were drawn using the MicroCal Origin Program.

activity was determined as $280 \mathrm{unit} / \mathrm{mg}$ at substrate concentrations of $2 \mathrm{mM}$ dihydrolipoamide and $3 \mathrm{mM} \mathrm{NAD}^{+}$ at $37^{\circ} \mathrm{C}$. This value was about 2.2-fold lower than that of normal human E3 activity that was determined for the same condition.

Due to FAD, normal E3 has characteristic UV-visible absorption spectrum patterns of flavoproteins from $350 \mathrm{~nm}$ to $550 \mathrm{~nm}$. The spectrum of normal E3 has two peaks at $455 \mathrm{~nm}$ and around $365 \mathrm{~nm}$ (Fig. 1, dot line). To detect any changes in the UV-visible absorption spectrum pattern of the mutant E3 that occurred by the substitution, the UV-visible spectrum of the mutant E3 was recorded as described in Materials and Methods. As shown in Fig. 1, the UV-visible spectrum of the mutant (solid line) was slightly different from that of the normal enzyme (dot line). The mutant's valley (around $396 \mathrm{~nm}$ ) was deeper than that of the normal enzyme. The ratio value between the mutant's peak $(455 \mathrm{~nm})$ and valley $(396 \mathrm{~nm})$ was determined as 6.8 , while that of the normal enzyme was 3.7. This increased ratio value might indicate a more hydrophobic microenvironment at the FAD binding region of the mutant E3 since the ratio value that was observed in the UV-visible spectrum of FAD alone in the hydrophilic buffer solution was around 1.7. These changes in the ratio values between the peak and valley was not observed in the other mutant E3s (Kim, 1999a, b). No amino acid was modified at the active site disulfide bond region. The ratio value between the maximum absorbance value, which was around $458 \mathrm{~nm}$ due to FAD and that around $274 \mathrm{~nm}$ due to aromatic amino acid residues, is a good indicator for E3 purity. The ratio value was determined as 6.6 , indicating that the purity of the mutant was very high.

Fluorescence is very sensitive to conformational changes, ligand bindings, and molecular interactions in proteins (Freifelder, 1982). When human E3s were excited at $296 \mathrm{~nm}$, 


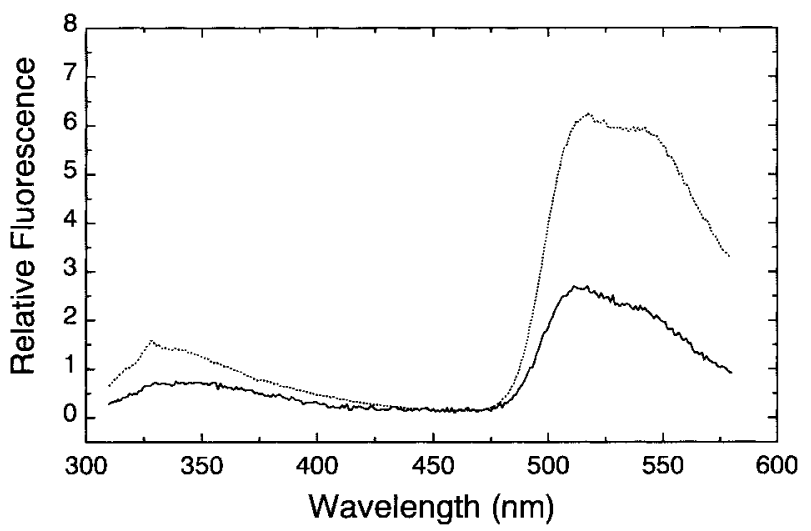

Fig. 2. Fluorescence spectra of the mutant $(7.6 \mu \mathrm{M}$, solid line) and wild-type $(12.5 \mu \mathrm{M}$, dot line) recombinant human E3s. The enzymes were excited at $296 \mathrm{~nm}$, and the emissions were observed from $302 \mathrm{~nm}$ to $580 \mathrm{~nm}$. The data were transferred to an ASCII file. The spectra were drawn using the MicroCal Origin Program.

two fluorescence emissions were observed in both mutant and normal enzymes (Fig. 2). The first emission was from $310 \mathrm{~nm}$ to $370 \mathrm{~nm}$, due to aromatic amino acids; the second emission was from $480 \mathrm{~nm}$ to over $580 \mathrm{~nm}$, due to FAD. The spectrum patterns of both mutant and normal E3s were similar considering their concentration differences. Only a slight difference was observed in the second emission, indicating small changes in the FAD environment in the mutant E3.

In the known structure of Azotobacter vinelandii E3, the hydroxyl group of Thr may interact with the adenosine phosphate of FAD (Mattevi et al., 1991). The difference between the two amino acids, Thr and Val, is that Thr has a hydroxyl group $(-\mathrm{OH})$ while Val has a methyl group $\left(-\mathrm{CH}_{3}\right)$ at their side chains. The hydroxyl group can interact with the adenosine phosphate of FAD, but the methyl group cannot. Three may be a slight size difference between the two amino acids. The substituted methyl group provides more hydrophobic microenvironment at the FAD binding region than the hydroxyl group does. These factors may result in the slight difference that is observed in the UV-visible and fluorescence spectra of the mutant E3.

As expected, the substitution of Thr-44 to Val in human E3 did not result in dramatic changes in the human E3 structure and function. However, from this study, the following reasons for the Thr conservation in the human E3, and possibly many other E3s, can be suggested. (1) The Thr-44 of the human E3 is important for the proper microenvironment at the FAD binding region by presumably making similar interactions with the adenosine phosphate of FAD, as observed in the three-dimensional structure of Azotobacter vinelandii E3, and (or) providing a more hydrophilic microenvironment at the FAD binding region. (2) This proper microenvironment at the FAD binding region may account for the approximate 2.2-fold efficient E3 activity of the normal human E3.
Acknowledgments The author thanks Dr. Mulchand S. Patel (State University of New York, Buffalo) for the generous gift of an E. coli XL1-Blue that contained a human E3 expression vector. The author is grateful to Dr. Tai Jong Kang (Daegu University) for providing a fluorometer.

\section{References}

Carothers, D. J., Pons, G. and Patel, M. S. (1989) Dihydrolipoamide dehydrogenase: functional similarities and divergent evolution of the pyridine nucleotide-disulphide oxidoreductases. Arch. Biochem. Biophys. 268, 409-425.

Cho, Y. -J. (2000) The carboxyl terminal amino acid residues glutamine 276-threonine 277 are important for actin affinity of the unacetylated smooth $\alpha$-tropomyosin. J. Biochem. Mol. Biol. 33, 531-536.

Freifelder, D. (1982) Physical Biochemistry, 2nd ed., W. H. Freeman and Company, New York, New York.

Jentoft, J. E., Shoham, M., Hurst, D. and Patel, M. S. (1992) A structural model for human dihydrolipoamide dehydrogenase. Proteins: Struct. Funct. Genet. 14, 88-101.

Kim, H. (1999a) Protein engineering of an artificial intersubunit disulfide bond linkage in human dihydrolipoamide dehydrogenase. J. Biochem. Mol. Biol. 32, 76-81.

Kim, H. (1999b) Deletion of the last five amino acid residues in human dihydrolipoamide dehydrogenase. Bull. Korean Chem. Soc. 20, 1221-1224.

Kim, H. (2001) Consensus sequence for the active site disulfide bond region of dihydrolipoamide dehydrogenases with known primary structures. J. Nat. Sci. 18, 23-29.

Kim, H., Liu, T.-C. and Patel, M. S. (1991) Expression of cDNA sequences encoding mature and precursor forms of human dihydrolipoamide dehydrogenase in Escherichia coli. J. Biol. Chem. 266, 9367-9373.

Kim, H. and Patel, M. S. (1992) Characterization of two sitespecifically mutated human dihydrolipoamide dehydrogenases (His-452 -> Gln and Glu-457 -> Gln). J. Biol. Chem. 267, 5128-5132.

Kim, S. I., Kim, S. -J., Leem, S. -H., Oh, K. -H. and Park, Y. -M. (2001) Site-Directed Mutagenesis of Two Cysteines $(155,202)$ in Catechol 1,2-dioxygenase $\mathrm{I}_{1}$ of Acinetobacter lwoffii K24. J. Biochem. Mol. Biol. 34, 172-175.

Lee, Y. -S. and Cho, Y. -D. (2001) Mutation of cysteine-115 to alanine in Nicotiana glutinosa orinithine decarboxylase reduces enzyme activity. J. Biochem. Mol. Biol. 34, 472-477.

Liu, T., Korotchkina, L. G., Hyatt, S. L., Vettakkorumakankav, N. N. and Patel, M. S. (1995) Spectroscopic studies of the characterization of recombinant human dihydrolipoamide dehydrogenase and its site-directed mutants. J. Biol. Chem. 270, 15545-15550.

Mattevi, A., Obmolova, G., Sokatch, J. R., Betzel, C. and Hol, W. G. J. (1992) The refined crystal structure of Pseudomonas putida lipoamide dehydrogenase complexed with $\mathrm{NAD}^{+}$at 2.45 $\AA$ resolution. Proteins; Struct. Funct. Genet. 13, 336-351.

Mattevi, A., Schierbeek, A. J. and Hol, W. G. J. (1991) The refined crystal structure of Azotobacter vinelandii lipoamide dehydrogenase at 2.2 A resolution. J. Mol. Biol. 220, 975-994

Pai, E. F. and Schulz, G. E. (1983) The catalytic mechanism of glutathione reductase as derived from X-ray diffraction analyses 
of reaction intermediates. J. Biol. Chem. 258, 1752-1757.

Pons, G., Raefsky-Estrin, C., Catothers, D. J., Pepin, R. A., Javed, A. A., Jesse, B. W., Ganapathi, M. K., Samols, D. and Patel, M. S. (1988) Cloning and cDNA sequence of the dihydrolipoamide dehydrogenase component of human ketoacid dehydrogenase complexes. Proc. Natl. Acad. Sci. USA 85, 1422-1426.

Reed, L. J. (1974) Multienzyme complexes. Acc. Chem. Res. 7, 40-46.

Schierbeek, A. J., Swarte, M. B. A., Dijkstra, B. W., Vriend, G., Reed, R. J., Hol, W. G. J. and Drenth, J. (1989) X-ray structure of lipoamide dehydrogenase from Azotobacter vinelandii determined by a combination of molecular and isomorphous replacement techniques. J. Mol. Biol. 206, 365-
379.

Thekkumkara, T. J., Pons, G., Mitroo, S., Jentoft, J. E. and Patel, M. S. (1989) Molecular biology of the human pyruvate dehydrogenase complex: structural aspects of the E2 and E3 components. Ann. N. Y. Acad. Sci. 573, 113-129.

Thieme, R., Pai, E. F., Schirmer, R. H. and Schulz, G. E. (1981) Three-dimensional structure of glutathione reductase at $2 \AA$ resolution. J. Mol. Biol. 152, 763-782.

Walker, J. L. and Oliver, D. J. (1986) Glycine decarboxylase multienzyme complex. Purification and partial characterization from leaf mitochondria. J. Biol. Chem. 261, 2214-2221.

Williams, C. H., Jr. (1976) Flavin containing dehydrogenases: in Enzymes (Boyer, P., ed.), 3rd ed., Vol. 13, 89-173, Academic Press, New York, New York. 